\title{
The Design of an Evaluation Framework for Diabetes Self-Management Education and Support Programs Delivered Nationally
}

Jenny Louise Olson ( $\square$ jenny.olson@diabeteswa.com.au )

Diabetes WA https://orcid.org/0000-0003-2777-3794

\section{Becky White}

Diabetes WA

\section{Helen Mitchell}

Diabetes WA

\section{Jennifer Halliday}

The Australian Centre for Behavioural Research in Diabetes

\section{Timothy Skinner}

The Australian Centre for Behavioural Research in Diabetes

\section{Deborah Schofield}

Diabetes WA

\section{Jennifer Sweeting}

Diabetes WA

Natasha Watson

Diabetes WA

\section{Methodology}

Keywords: implementation science, program evaluation, diabetes self-management education and support, health services research, self-management, chronic disease, diabetes mellitus, selfdetermination, psychosocial adjustment

Posted Date: March 10th, 2021

DOl: https://doi.org/10.21203/rs.3.rs-264927/v1

License: (c) (i) This work is licensed under a Creative Commons Attribution 4.0 International License.

Read Full License 


\section{Abstract \\ Background}

The aim of this work was to develop a National Evaluation Framework to facilitate the standardization of delivery, quality, reporting, and evaluation of diabetes education and support programs delivered throughout Australia through the National Diabetes Services Scheme (NDSS). The NDSS is funded by the Australian Government, and provides access to diabetes information, education, support, and subsidized product across diverse settings in each state and territory of Australia through seven independent serviceproviders. This article reports the approach undertaken to develop the Framework.

\section{Methods}

A participatory approach was undertaken, focused on adopting nationally consistent outcomes and indicators, nominating objectives and measurement tools, specifying evaluation processes, and developing quality standards. Existing programs were classified based on related, overarching indicators enabling the adoption of a tiered system of evaluation.

\section{Results}

Two outcomes (i.e., improved clinical, reduced cost) and four indicators (i.e., improved knowledge and understanding, self-management, self-determination, psychosocial adjustment) were adopted from the Eigenmann and Colagiuri national consensus position statement for diabetes education. This allowed for the identification of objectives (i.e., improved empowerment, reduced distress, autonomy supportive program delivery, consumer satisfaction) and related measurement instruments. Programs were categorized as comprehensive, topic-specific, or basic education, with comprehensive programs allocated to receive the highest-level of evaluation. Twelve quality standards were developed, with existing programs tested against those standards. Based on the results of testing, two comprehensive (OzDAFNE for people with type 1 diabetes, DESMOND for people with type 2 diabetes), and eight topic-specific (CarbSmart, ShopSmart, MonitorSmart, FootSmart, MedSmart, Living with Insulin, Insulin Pump Workshop, Ready Set Go - Let's Move) structured diabetes self-management education and support programs were nominated for national delivery.

\section{Conclusions}

The National Evaluation Framework has facilitated consistency of program quality, delivery, and evaluation of programs delivered by multiple service providers across diverse contexts. The Framework could be applied by other service providers who facilitate multiple diabetes education and support 
programs and could be adapted for use in other chronic disease populations where education and support are indicated.

\section{Contributions To The Literature}

- This study describes the collaborative processes undertaken to develop a National Evaluation Framework for diabetes self-management education and support programs delivered via the Australian Government-funded National Diabetes Service Scheme.

- The Framework is evidence-based and includes standardized outcomes, indicators, objectives, instruments, quality standards, program categories, and a tiered system of evaluation.

- The Framework has facilitated standardization of program quality, delivery, and evaluation across multiple service providers in varying contexts across Australia.

- The Framework could be adopted for use in other regions or for application in other chronic disease populations for whom health education and support is indicated.

\section{Background}

Diabetes is a chronic condition characterized by elevated blood glucose levels and is associated with increased risk of serious health complications (1). It is estimated that more than 451-million adults are living with diabetes globally, with projections indicating this could increase to 693-million by 2024 (2). Approximately 5-million deaths were attributed to the condition globally during 2017 (2). Diabetes also results in significant financial burden, with related global healthcare expenditure estimated at USD\$850 billion annually (2). Diabetes self-management education and support programs (DSMES) provided by consumer health organizations and health professionals have the potential to significantly improve a person's ability to live well with diabetes (3), and reduce risk of diabetes-related complications and health system burden (4). Given the rising prevalence of diabetes and the large financial burden attributed to the condition, the need to evaluate the outcomes of programs implemented in community settings has never been more paramount.

The Australian Government invests significantly in diabetes-related services and care. Since 1987, programs, services, and access to diabetes product has been facilitated through the National Diabetes Services Scheme (NDSS), an initiative of the Australian Government, administered by Diabetes Australia (5). The aim of the NDSS is to enhance the capacity of people with diabetes and to live a life in which the impact of diabetes is minimized. Seven state and territory Agents (herein referred to as 'Agents') receive funding through the NDSS to support people living with diabetes, across diverse settings. As with many national programs for health service delivery, the types of programs and services offered, the objectives targeted within programs, and the evaluation processes employed by individual Agents varied extensively. This heterogeneity across organizations and programs impeded consistent evaluation of the reach, outcomes, and impact of the NDSS. 
Some programs offered through the NDSS are backed by extensive evidence garnered from research studies. These included Dose Adjustment or Normal Eating (DAFNE; with the Australian edition entitled 'OzDAFNE') for people with type 1 diabetes (6-10); and Diabetes Education and Self-Management for Ongoing and Newly Diagnosed (DESMOND) for people with type 2 diabetes (11-14). However, evidence of the effectiveness of many other programs offered through the NDSS was limited, as was evaluation of the outcomes of DESMOND and OzDAFNE delivered across service providers and diverse contexts nationally. Without direct and consistent national evaluation of program outcomes, the broader real-world impact of NDSS programs was unclear. Given the substantive expenditure of public funds, it is important to demonstrate program outcomes and impact, and continually assess and improve the quality of services provided. Thus, the need for a standardized national approach to evaluation of programs facilitated through the NDSS was identified. A recent policy shift by the Australian Government to outcome-based models and funding further reinforced the need for systematic evaluation of NDSS programs.

The aim of this article is to describe the process undertaken to develop and implement a National Evaluation Framework for NDSS education and support programs in Australia. To our knowledge, this is the first framework for diabetes self-management education that describes the process of standardization of DSMES and evaluation processes at a national level.

\section{Methods}

\section{Aim and Design}

The aim of this work was to systematically develop a National Evaluation Framework to: (a) facilitate national standardization of diabetes programs and evaluation throughout Australia, (b) ensure consistent program quality and reporting by NDSS service providers, and (c) facilitate consistent evaluation processes and reporting of overall program outcomes. A participatory approach was adopted to achieve these aims, with two key areas of focus. This approach is depicted in Figure 1 and described in further detail below.

\section{Participatory Approach}

A National Evaluation Team (NET) was established to lead the development and ongoing implementation of the National Evaluation Framework. The team was comprised of research and evaluation experts employed by Diabetes WA, with funding support from the NDSS. The Team met regularly with representatives from Diabetes Australia, who provided project governance and oversight.

An Expert Reference Group (ERG) was established to guide the design, development, and implementation of the Framework. The group included representatives from Diabetes Australia, the Australian Centre for Behavioural Research in Diabetes, the Australian Diabetes Educators of Australia, Deakin University, Charles Darwin University, a consumer representative, and Agents. 
The NET and ERG consulted widely with the NDSS National Services Group, a diverse group of health professionals working with people with diabetes (e.g., Dietitians, Diabetes Educators, and Health Service Managers) representing Agents. The range of health professionals providing diabetes services in Australia is diverse, both organizationally and geographically. Therefore, understanding the viewpoints of a broad range of experts was integral in shaping the design and implementation of the Framework.

\section{Nationally Standardized Outcomes and Indicators, Program Categories, Objectives and Measurement Tools, and Evaluation Processes}

Research evidence guided the identification of outcomes, indicators, and objectives for diabetes programs and services that were most likely to lead to favorable outcomes. To support nationally consistent program delivery and evaluation and ensure the best opportunity to improve the outcomes of people with diabetes, outcomes and indicators were adopted from the national consensus position statement previously developed on behalf of Diabetes Australia (15). Informed by an international evidence-base and extensive consultation, the position statement outlines three key goals for diabetes education, including: (1) optimal adjustment to living with diabetes, (2) optimal health outcomes, and (3) optimal cost effectiveness. Goals 2 and 3 recognize the potential impact of diabetes education on physical health outcomes and optimal cost effectiveness. However, the statement acknowledges that it is challenging to directly attribute these to diabetes education, and thus, attention should primarily focus on Goal 1. Components or 'indicators' of diabetes education identified as relating to the goal of optimal adjustment to living with diabetes include: (a) knowledge and understanding, (b) self-management (i.e., diabetes self-care skills and behaviors), (c) self-determination and (d) psychological adjustment.

The identification of outcomes and indicators for DSMES allowed for the categorization of existing diabetes programs based on the indicators targeted within those programs. This required the identification of programs currently delivered throughout Australia, and the collection of information related to those programs, such as targeted population (e.g., people with type 1 diabetes) and outcomes, and program duration. Agents reported this information to the NET. The information was then collated to form a database of programs. The targeted outcomes of each program were compared against the outcomes and indicators specified in the National Evaluation Framework. Programs were then grouped into categories, based on the outcomes targeted within those programs.

The categorization of programs enabled the specification of a tiered system of evaluation. Each category of programs was ascribed to an individual tier of evaluation, with specific evaluation outputs allocated to the programs within each tier. More comprehensive evaluation processes were ascribed to resourceintensive, higher-cost programs predicted to demonstrate the greatest behavioral impact. Program objectives were defined, and appropriate measurement tools selected to assess outcomes against those objectives. Potential participant burden was weighed against the benefits of intensive program 
evaluation. Moreover, pragmatic assessment of program objectives had to be applied across the broad range of programs being delivered.

The selection of measurement instruments was guided by a recent review of psychometric tools for diabetes education services (19). The review investigated instruments to measure commonly targeted outcomes of diabetes education. Instruments were assessed on suitability, validity, reliability, feasibility, and sensitivity. Just three of the 37 tools evaluated met all five criteria. Several other instruments were deemed suitable as they met all but one of the criteria. Based on these findings, potential instruments to measure targeted objectives were selected for consideration by the ERG. Specific instruments were then nominated for inclusion in the National Evaluation Framework.

\section{Quality Standards and Assessment}

A set of quality standards was developed to ensure that NDSS programs were of high-quality and contained components to elicit key outcomes associated with optimal diabetes self-management. DSMES can have a significant positive effect on health (16). For example, DSMES are effective in reducing blood glucose levels and psychosocial outcomes in people with type 2 diabetes (17-19) and type 1 diabetes (17). Such programs have also been assessed as cost effective in empowering people with diabetes to self-manage their condition and mitigate risks of complications, with an incremental cost-effectiveness ratio of USD \$5,047 per additional quality-adjusted life year, when compared to usual care (20). Therefore, it was important that the quality standards supported the delivery of programs that were structured and tailored to support the needs of the individual.

The content of the quality standards was informed by existing national and international standards and guidelines (21-24), state and federal government policy for primary care, chronic conditions, and diabetes $(25,26)$, and the National Safety and Quality Health Service Standards (NSQHS; 27). Collectively, these standards and guidelines recommend that DSMES should be person-centered (i.e., responsive to the unique needs of the individual), and provided at the time of diagnosis and throughout the person's journey with diabetes. It is also recommended that DSMES should be accessible, culturally appropriate, and provide appropriate information and education for all people with diabetes, their families, and carers. The importance of strategies promoting active learning, goal setting, and supported decision making is also recognized. Programs should have a written curriculum, standardized facilitator training, and a quality development pathway to ensure fidelity and facilitate quality assurance.

Existing topic specific and comprehensive NDSS DSMES were assessed in accordance with the newly developed standards, to ensure consistent quality across all states and territories. Behavior change outcomes were not expected from tier 1 basic education programs, thus no formal review of these programs was undertaken; however, the standards provide a general guide for the provision of basic education programs through the NDSS. 
In September 2016, representatives of each Agent assessed their existing DSMES utilizing a user-friendly self-assessment tool, developed to guide the application of the quality standards into practice. The tool is presented in Appendix A. To ensure internal validity, the evaluations of DSMES conducted by each of the Agents were then independently reviewed by two members of the NET. The reviewers then met to discuss the outcomes of the independent assessments and whether individual programs met quality standards. Discrepancies between reviewers were identified and resolved through mutual agreement and feedback, including areas for quality improvement, was provided to the NDSS Agents.

\section{Results}

A National Evaluation Framework for diabetes education and support programs facilitated through the NDSS was developed. The Framework consists of nationally standardized outcomes and indicators, program categories, objectives, measurement tools, evaluation processes, and quality standards. These are summarized in Fig. 2.

\section{Outcomes and Indicators, Program Categories, Objectives and Measurement Tools, and Evaluation Processes}

\section{Outcomes and Indicators}

Four indicators (i.e., improved knowledge and understanding, self-management, self-determination, and psychological adjustment) and two outcomes (i.e., improved clinical and reduced cost) derived from the National Consensus Position Statement (15) were included in the NDSS National Evaluation Framework. These outcomes and indicators represent the aims of DSMES facilitated throughout Australia via the NDSS.

\section{Program Categories}

Programs were classified into three distinct categories. Low intensity, less structured programs targeting knowledge and understanding and delivered to large groups, were classified as 'basic education'. These programs were characterized as high-reach and low-cost, and did not incorporate behavioral strategies to foster improvements in self-management, self-determination, or psychological adjustment. Thus, while basic education programs aimed to improve knowledge and understanding of diabetes, these programs were assessed as unlikely to elicit behavior change associated with improved clinical and cost outcomes.

More intensive, targeted DSMES focusing on a specific diabetes self-management topic (e.g., foot care) and covering at least one indicator of the Framework in addition to knowledge and understanding were classified as 'topic specific'. Topic specific DSMES were short in duration (e.g., 3-hours), delivered in smaller groups, and anticipated to be higher cost with lower reach than basic education programs. However, these structured programs were anticipated to foster some degree of behavior change through strategies to improve self-management, self-determination, and/or psychological adjustment, in addition to increasing knowledge and understanding. 
Group DSMES that were of longer duration, provided structured self-management education covering a range of diabetes-related topics, and targeted all four indictors were classified as 'comprehensive'. Comprehensive DSMES are highly structured and resource intensive, with higher estimated cost per participant and lower reach than programs classified at other levels. However, such programs were anticipated to be the most effective in eliciting behavioral change, with the inclusion of multiple strategies to support improved self-management, self-determination, psychological adjustment, and knowledge and understanding.

\section{Objectives and Measurement Tools}

The burden of measuring constructs related to all four indicators was deemed too great and the assessment of constructs related to self-determination and psychological adjustment were prioritized. Moreover, direct evaluation of clinical outcomes and cost was not feasible, given the available resources, accessibility of information, and scope of the NDSS (e.g., Agents do not have access to medical records for evaluation of clinical outcomes). Likewise, intensive longitudinal surveillance necessary to determine cost savings would require a substantial financial investment beyond current levels of resourcing and access to information not currently available to Agents or evaluators.

Four objectives related to self-determination and psychological adjustment were identified for DSMES, including: (a) increased diabetes-related empowerment, (b) participant perceptions that facilitators were autonomy supportive, (c) reduced diabetes-related distress, and (d) positive consumer satisfaction. Four instruments were selected to measure outcomes against these objectives.

Increased Diabetes-Related Empowerment. Diabetes-related empowerment refers to an individual's perceived psychosocial self-efficacy to manage diabetes (28), and is positively associated with diabetes knowledge, medication adherence, and self-care behaviors (29). The diabetes empowerment scale - short form (DES-SF) (28) was selected to measure this construct. The DES-SF provides a reliable 8-item measure of diabetes-related psychosocial self-efficacy $(\alpha=0.84)$. Items are statements reflecting beliefs of empowerment and confidence to manage diabetes (e.g., "I can make choices about my diabetes management that are right for me."). Responses are collected on 5-point scales ( 1 = strongly disagree and 5 = strongly agree). Higher scores indicate greater diabetes-related empowerment.

Perceptions of Autonomy Support. The health care climate questionnaire (HCCQ; 30) was selected to evaluate a construct related to the indicator of self-determination. Perceptions of autonomy support from health care providers have been associated with significant improvements in autonomous motivation for glucose control and reductions in $\mathrm{HbA} 1 \mathrm{c}$ among people receiving treatment for diabetes (31). Moreover, facilitator delivery style may influence the effectiveness of DSMES (32). Inclusion of the HCCQ provided a mechanism of monitoring the fidelity of program delivery by educators specifically trained in a personcentered approach. The HCCQ demonstrates high internal reliability $(a=0.95)(30)$. A modified 10 -item version of the scale was applied, including the items most relevant to the person-centered delivery style associated with effective DSMES $(a=0.76)$. Items are statements reflecting perceived autonomy support (e.g., I feel that the staff has provided me with choices and options." Responses are collected on 5-point 
scales $(1=$ not true at all and $5=$ very true $)$. Higher scores represent greater perceived autonomy support from health care providers.

Reduced Diabetes-Distress. Diabetes distress refers to psychosocial distress specifically related to the burden of living with, and managing, diabetes and its complications (33). Diabetes distress is highly prevalent and associated with sub-optimal self-care and poorer emotional well-being (34). The 20-item Problem Areas in Diabetes Scale (PAID; 35) was selected to measure diabetes distress. Items build on the stem "Which of the following diabetes issues are currently a problem for you?" (e.g., "Feeling discouraged with your diabetes treatment plan"). Responses are collected on a 5-point Likert scale $(0=$ not a problem and $4=$ serious problem). Higher scores indicate greater diabetes-related distress. The scale demonstrates high internal reliability $(a=0.95)$ and concurrent validity has been established (35).

Consumer Satisfaction. A global measure of consumer satisfaction was also adopted. Net Promotor Score (NPS; 36,37$)$ reflects the proportion of participants likely to recommend a program to others (i.e., promoters), minus the proportion of participants defined as detractors (i.e., those not likely to recommend the program). NPS consists of a single item, with responses collected on an 11-point scale $(0=$ not at all likely to recommend to others and $10=$ extremely likely to recommend to others). The NPS score is calculated by subtracting the proportion of those who responded from $0-6$ on the scale ('detractors') from the proportion who responded 9-10 ('promoters'). Higher NPS scores indicate greater participant satisfaction. NPS scores are compared against international health industry averages, as reflected in the International NPS \& CX Benchmarks report (37).

\section{Evaluation Processes}

Basic education programs were nominated at the first tier of evaluation. Evaluation focused on knowledge and understanding, and consumer satisfaction. As basic programs aim only to increase knowledge and understanding, with no focus on behavioral change, evaluation of tier one programs excluded evaluation of diabetes empowerment, distress, and autonomy support, and measures were administered immediately post-program.

DSMES classified as topic specific were ascribed to the second tier of evaluation. Evaluation included assessment of knowledge and understanding, confidence for self-management, self-determination, and psychological adjustment. As behavioral change was anticipated from DSMES within this tier, data collection was planned to take place pre- and post-program participation to assess changes in outcome measures.

DSMES classified as comprehensive were nominated to the third, and highest, evaluation tier. More complex evaluation was planned, with assessment of all behavior change objectives relating to diabetes empowerment and diabetes distress. Data collection was planned for three time points including pre- and post-program, and follow-up (e.g.at 3- or 12-months). The final NDSS programs included within each category and the ascribed evaluation tiers are presented in Table 1. 


\section{Quality Standards and Assessment}

\section{Quality Standards}

Eight quality standards were developed for DSMES (Table 2). It was expected that programs meeting these standards would be more likely to achieve the outcomes and indicators adopted in the National Evaluation Framework compared to programs that do not meet the standards.

\section{Assessment}

The assessment and review of existing programs resulted in the identification of a suite of programs meeting the quality standards. These programs were recommended and subsequently nominated for national delivery by the NDSS administrator, Diabetes Australia. Programs included two comprehensive DSMES - OzDAFNE, for people with type 1 diabetes, and DESMOND for people with type 2 diabetes. Five topic specific programs met the quality standards, including programs related to self-management of carbohydrate intake (CarbSmart), food shopping and interpreting nutrition labels (ShopSmart), glucose monitoring (MonitorSmart), foot care (FootSmart), and managing medication (MedSmart).

Gaps in service provision were then noted, including a lack of programs to support physical activity, selfmanagement of insulin and the use of insulin pumps. To fill this gap, two programs initially assessed as not meeting the standards went through a quality improvement process and were subsequently reassessed, resulting in inclusion in the national program suite. A new program, 'Ready Set Go - Let's Move' was also developed to support people with diabetes to safely participate in physical activity. All other programs that did not meet the requisite quality standards were no longer supported for delivery through the NDSS.

\section{Discussion}

This National Evaluation Framework has led to nationally consistent delivery of evidence based, personcentered DSMES, including standardized curriculum and facilitator training. The Framework was fully implemented across all NDSS diabetes education and support programs by 2017. Data collection and analysis continue, to facilitate ongoing evaluation and quality improvement.

As health care costs continue to increase, government-funded organizations are under increasing pressure to demonstrate that programs and services are cost-effective, impactful, and achieve targeted outcomes. Effective evaluation of programs and services and transparency in the expenditure of public funds is critical. The National Evaluation Framework helps to maintain accountability in terms of the justification of government spending, and importantly, to people in Australia living with diabetes. 
The Framework represents an innovative and comprehensive approach to achieving national consistency in quality and delivery and evaluation of DSMES. The methods described in this article may guide serviceproviders in other regions around the world, and those providing services for other clinical populations, or other chronic conditions that have a significant requirement for self-management support. Moreover, the Framework could be implemented or adapted for use by other service providers who administer multiple diabetes education programs across a range of settings.

A participatory approach involving service providers, scheme administrators, and evaluation professionals resulted in consensus around the National Evaluation Framework within the NDSS. The Framework provided for the categorization of the diabetes education and support programs available nationally through the NDSS, and guides evaluation processes based on targeted indicators. Moreover, for the first time in Australia, quality standards for diabetes education and support facilitated through the Australian Government-funded NDSS were developed and implemented. These standards ensure that all NDSS programs are of the highest quality, are person-centered, and contain key aspects known to be associated with optimal consumer outcomes, in addition to ensuring that programs comply with the Australian NSQHS.

\section{Strengths, Limitations, and Future Directions}

The approach taken to develop the Framework was collaborative, systematic, and informed by evidence. The establishment and funding of a NET was critical to the development and implementation of the Framework and represents a clear strength of this work. Furthermore, the continued resourcing of a team of specialized evaluation experts ensures ongoing implementation and refinement of the Framework, thereby allowing for continued critical assessment and quality improvement of Australian Governmentfunded NDSS programs.

The development of the Framework involved extensive consultation with stakeholders to ensure acceptability among NDSS administrators and Agents. The credibility of the Framework and the process was enhanced by the contributions of the ERG who contributed extensive experience in diabetes care. The establishment and implementation of common objectives and quality standards supports nationally consistent delivery of high-quality programs targeting factors known to be associated with improved outcomes among people with diabetes by service providers across all states and territories of Australia. Moreover, the application of standardized evaluation processes utilizing evidence-based instruments for the assessment of outcomes ensures accountability and transparency in service delivery and expenditure of public funds.

Despite the strengths of this work, there are limitations that should be acknowledged. Firstly, greater input from Australians with diabetes could have allowed for the identification of additional factors to be considered when developing the Framework. Secondly, the benefits of rigorous and detailed program evaluation need to be balanced against the availability of resources, accessibility of information, and the need to ensure people who take part in the programs are not overburdened. Based on the available 
evidence, it is anticipated that the provision of DSMES that improve knowledge and understanding, selfmanagement, self-determination, and psychosocial adjustment will lead to improved clinical outcomes and reduced economic burden at the individual and societal level (15). However, direct evaluation of these outcomes is not feasible given resources, readily available information, and the interests of NDSS consumers. Moreover, the objectives, quality standards, and measurement instruments were developed or selected based on their suitability to the general population of people with diabetes and may not be suitable to all sub-populations (e.g., culturally and linguistically diverse, Aboriginal and Torres Strait Islanders). The development and refinement of national evaluation tools suitable for application within these populations remains a priority.

\section{Conclusions}

The development of the National Evaluation Framework, including specification of key outcomes, indicators, objectives and tools, and evaluation processes, utilized a systematic and collaborative approach. The development and implementation of quality standards ensured that NDSS programs are nationally consistent, high quality, and target objectives known to be associated with optimal diabetes self-management, improved clinical outcomes, and reduced financial burden. Ongoing assessment of the effectiveness of NDSS programs facilitates the identification of programs achieving desired outcomes and provides opportunities for quality improvement. The data generated through the implementation of the Framework informs service planning and delivery, ensuring optimal impact on the health and wellbeing of people with diabetes, and a high degree of accountability in the expenditure of public funds.

\section{List Of Abbreviations}

DAFNE: Dose Adjustment for Normal Eating

DESMOND: Diabetes Education and Self-Management for Ongoing and Newly Diagnosed

DES-SF Diabetes Empowerment Scale - short form

DSMES: Diabetes self-management education and support

ERG: Expert Reference Group

HCCQ: Health Care Climate Questionnaire

NDSS: National Diabetes Services Scheme

NDSS Agents: State and Territory Agents delivering services on behalf of the NDSS

NET: National Evaluation Team

NPS: Net Promotor Score 
OzDAFNE: Dose Adjustment for Normal Eating (Australian edition)

PAID: Problem Areas in Diabetes Scale

\section{Declarations}

Ethics approval and consent to participate: Ethical approval and consent were not required to develop an Evaluation Framework for health-service provision.

Consent for publication: Not applicable

Availability of data and materials: Data sharing is not applicable as no datasets were generated or analyzed.

Competing interests: Diabetes WA was funded by the Australian Government Department of Health to develop and implement the National Evaluation Framework. Diabetes WA receives funding as an NDSS Agent, and to facilitate national NDSS evaluation. JO, NW, JS, and DS are employees of Diabetes WA. BW and HM were employees of Diabetes WA when much of the work was undertaken. JH and TS were members of the ERG who guided the work.

Funding: This work was funded by the Australian Government Department of Health, via the NDSS.

Authors' contributions: HM originally identified the need for a nationally consistent approach to evaluation of NDSS programs. NW led the development and implementation of the Framework. JO and BW led the development of the manuscripts. $\mathrm{HM}, \mathrm{JH}, \mathrm{TS}, \mathrm{DS}$, JS and NW revised the content and structure of the manuscripts. All authors read and approved the final manuscript.

Acknowledgements: The authors would like to acknowledge Eigenmann and Colagiuri (15) from the Australian Health Policy Institute, who developed the National Consensus Position for diabetes education on behalf of Diabetes Australia. The Framework of goals, outcomes, and indicator areas for diabetes education was then applied as the foundations of the NDSS National Evaluation Framework. The authors would also like to thank the members of the ERG who guided the application of the National Evaluation Framework, including representatives from Diabetes Australia, the Australian Centre for Behavioural Research in Diabetes (ACBRD), the Australian Diabetes Educators of Australia (ADEA), NDSS Agents, Deakin University, and Charles Darwin University, and the consumer representative. We would also like to thank members of the NDSS National Services Group for the feedback they provided.

\section{References}

1. World Health Organization. Diabetes 2020 [June 26, 2020]. Available from: https://www.who.int/health-topics/diabetes\#tab=tab_1.

2. Cho NH, Shaw JE, Karuranga S, Huang Y, da Rocha Fernandes JD, Ohlrogge AW, et al. IDF Diabetes Atlas: Global estimates of diabetes prevalence for 2017 and projections for 2045. Diabetes Res Clin 
Pract. 2018;138:271-81.

3. American Association of Diabetes Educators. An effective model of diabetes care and education: Revising the AADE7 self-care behaviors ${ }^{\circledR}$. The Diabetes Educator. 2020;46(2):139-60.

4. Chatterjee S, Davies MJ, Heller S, Speight J, Snoek FJ, Khunti K. Diabetes structured selfmanagement education programmes: a narrative review and current innovations. The lancet Diabetes endocrinology. 2018;6(2):130-42.

5. Diabetes Australia. About the NDSS Canberra2020 [Available from: https://www.ndss.com.au/aboutthe-ndss/.

6. Hopkins D, Lawrence I, Mansell P, Thompson G, Amiel S, Campbell M, et al. Improved biomedical and psychological outcomes 1 year after structured education in flexible insulin therapy for people with type 1 diabetes: the UK DAFNE experience. Diabetes Care. 2012;35(8):1638-42.

7. Speight J, Amiel S, Bradley C, Heller S, Oliver L, Roberts S, et al. Long-term biomedical and psychosocial outcomes following DAFNE (Dose Adjustment For Normal Eating) structured education to promote intensive insulin therapy in adults with sub-optimally controlled type 1 diabetes. Diabetes Res Clin Pract. 2010;89(1):22-9.

8. Kruger J, Brennan A, Thokala P, Basarir H, Jacques R, Elliott J, et al. The cost-effectiveness of the Dose Adjustment for Normal Eating (DAFNE) structured education programme: an update using the Sheffield Type 1 Diabetes Policy Model. Diabetic medicine. 2013;30(10):1236-44.

9. Speight J, Holmes-Truscott E, Harvey DM, Hendrieckx C, Hagger VL, Harris SE, et al. Structured type 1 diabetes education delivered in routine care in Australia reduces diabetes-related emergencies and severe diabetes-related distress: The OzDAFNE program. Diabetes Res Clin Pract. 2016;112:65-72.

10. Engel L, Cummins R. Impact of dose adjustment for normal eating in Australia (OzDAFNE) on subjective wellbeing, coping resources and negative affects in adults with type 1 diabetes: A prospective comparison study. diabetes research clinical practice. 2011;91(3):271-9.

11. Davies MJ, Heller S, Skinner TC, Campbell MJ, Carey ME, Cradock S, et al. Effectiveness of the diabetes education and self management for ongoing and newly diagnosed (DESMOND) programme for people with newly diagnosed type 2 diabetes: cluster randomised controlled trial. BMJ. 2008;336(7642):491.

12. Khunti K, Gray LJ, Skinner T, Carey ME, Realf K, Dallosso H, et al. Effectiveness of a diabetes education and self management programme (DESMOND) for people with newly diagnosed type 2 diabetes mellitus: three year follow-up of a cluster randomised controlled trial in primary care. BMJ: British Medical Journal. 2012;344(7860).

13. Gillett M, Dallosso H, Dixon S, Brennan A, Carey M, Campbell M, et al. The cost effectiveness of delivering the DESMOND Intervention (Diabetes Education and Self-Management for Ongoing and Newly Diagnosed) for people newly diagnosed with type 2 diabetes. BMJ. 2010;341:c4093.

14. Miller VM, Davies MJ, Etherton-Beer C, McGough S, Schofield D, Jensen JF, et al. Increasing patient activation through diabetes self-management education: Outcomes of DESMOND in regional Western Australia. Patient Educ Couns. 2020;103(4):848-53. 
15. Eigenmann CA, Colagiuri R. Outcomes and indicators for diabetes education - A national consensus position. Canberra; 2007.

16. Burke SD, Sherr D, Lipman RD. Partnering with diabetes educators to improve patient outcomes. Diabetes Metab Syndr Obes. 2014;7:45-53.

17. Hermanns N, Ehrmann D, Finke-Groene K, Kulzer B. Trends in diabetes self-management education: where are we coming from and where are we going? A narrative review. Diabet Med. 2020;37(3):43647.

18. Chrvala CA, Sherr D, Lipman RD. Diabetes self-management education for adults with type 2 diabetes mellitus: A systematic review of the effect on glycemic control. Patient Educ Couns. 2016;99(6):92643.

19. Olesen K, Folmann Hempler N, Drejer S, Valeur Baumgarten S, Stenov V. Impact of patient-centred diabetes self-management education targeting people with type 2 diabetes: an integrative review. Diabet Med. 2020;37(6):909-23.

20. Siegel KR, Ali MK, Zhou X, Ng BP, Jawanda S, Proia K, et al. Cost-effectiveness of interventions to manage diabetes: Has the evidence changed since 2008? Diabetes Care. 2020;43(7):1557.

21. International Diabetes Federation. Global IDF/ISPAD guideline for diabetes in childhood and adolescence. Brussels, Belgium; 2011.

22. International Diabetes Federation Guideline Development Group. Global guideline for type 2 diabetes. Diabetes Res Clin Pract. 2014;104(1):1.

23. Haas L, Maryniuk M, Beck J, Cox CE, Duker P, Edwards L, et al. National standards for diabetes selfmanagement education and support. The Diabetes Educator. 2012;38(5):619-29.

24. National Institute for Health and Care Excellence. Diabetes in adults 2016 [November 2, 2020]. Available from: https://www.nice.org.uk/guidance/qs6/chapter/quality-statement-2-structurededucation-programmes-for-adults-with-type-2-diabetes.

25. Department of Health Western Australia. Western Australian diabetes standards. Western Australia: Perth; 2014.

26. Department of Health Australia. Austraian national diabetes strategy 2016-2020. Canberra 2015.

27. Australian Commission on Safety and Quality in Healthcare. The National Safety and Quality Health Service Standards 2019 [January 25, 2021]. Available from: https://www.safetyandquality.gov.au/standards/nsqhs-standards.

28. Anderson RM, Funnell MM, Fitzgerald JT, Marrero DG. The Diabetes Empowerment Scale: a measure of psychosocial self-efficacy. Diabetes Care. 2000;23(6):739-43.

29. Hernandez-Tejada MA, Campbell JA, Walker RJ, Smalls BL, Davis KS, Egede LE. Diabetes Empowerment, Medication Adherence and Self-Care Behaviors in Adults with Type 2 Diabetes. Diabetes Technol Ther. 2012;14(7):630-4.

30. Williams G, Grow VM, Freedman ZR, Ryan RM, Deci EL. Motivational predictors of weight loss and weight-loss maintenance. J Personal Soc Psychol. 1996;70(1):115. 
31. Williams GC, Freedman ZR, Deci EL. Supporting autonomy to motivate patients With diabetes for glucose control. Diabetes Care. 1998;21(10):1644.

32. Skinner TC, Carey ME, Cradock S, Dallosso HM, Daly H, Davies MJ, et al. 'Educator talk' and patient change: some insights from the DESMOND (Diabetes Education and Self Management for Ongoing and Newly Diagnosed) randomized controlled trial. Diabetic medicine: a journal of the British Diabetic Association. 2008;25(9):1117-20.

33. Snoek FJ, Bremmer MA, Hermanns N. Constructs of depression and distress in diabetes: time for an appraisal. The Lancet Diabetes Endocrinology. 2015;3(6):450-60.

34. Skinner TC, Joensen L, Parkin T. Twenty-five years of diabetes distress research. Diabet Med. 2020;37(3):393-400.

35. Polonsky W, Anderson B, Lohrer PA, Welch G, Jacobson A, Aponte J, et al. Assessment of diabetes distress. Diabetes Care. 1995;18(6):754-60.

36. Reichheld F. The One Number you Need to Grow. Harvard business review. 2004;81:46-54, 124.

37. CustomerGauge. What is Net Promoter Score? 2020 [Available from: https://customergauge.com/net-promoter-score-nps/.

\section{Tables}




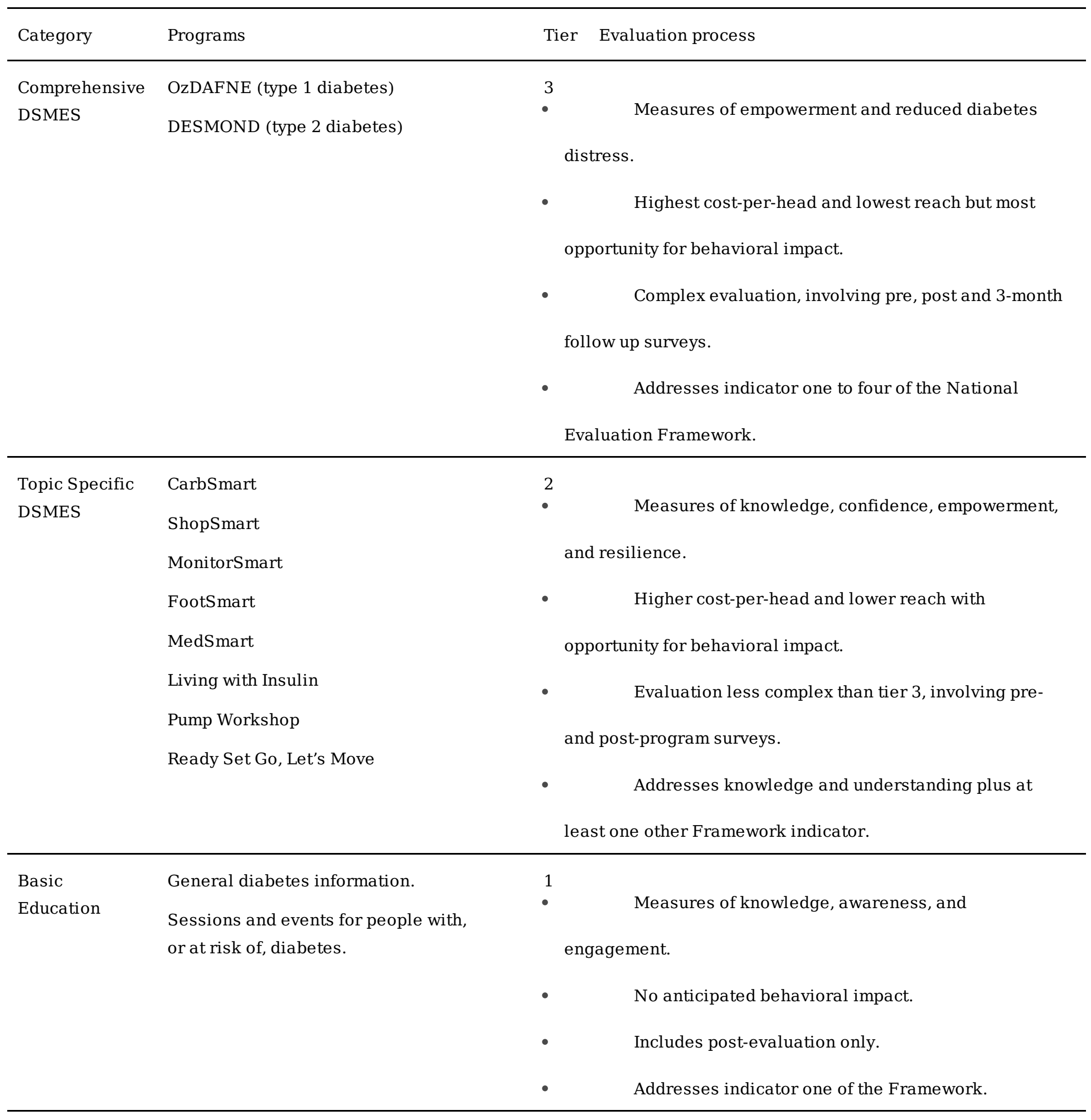

Table 2 Quality Standards for NDSS Diabetes Self-Management Education and Support 


\section{Standard Description}

1 Structured diabetes education should be offered to consumers as soon as possible after diagnosis and on an ongoing basis.

2 Structured diabetes education should be person-centered, use a variety of techniques to promote active learning, and be flexible enough to meet different needs.

3 Structured diabetes education should be provided by an appropriately trained facilitator.

4 Structured diabetes education should be evidence-based, reflect current clinical guidelines, and cover the four key indicators.

5

Structured diabetes education programs should strive to be equitable and accessible to all people.

6

Structured diabetes education should include a written curriculum with clearly defined learning aims and objectives.

7

Structured diabetes education content and resources must be assessed for and meet appropriate readability and health literacy levels.

8

Structured diabetes education should include evaluation that measures program aims and objectives, program fidelity and supports continuous quality improvement.

\section{Figures}

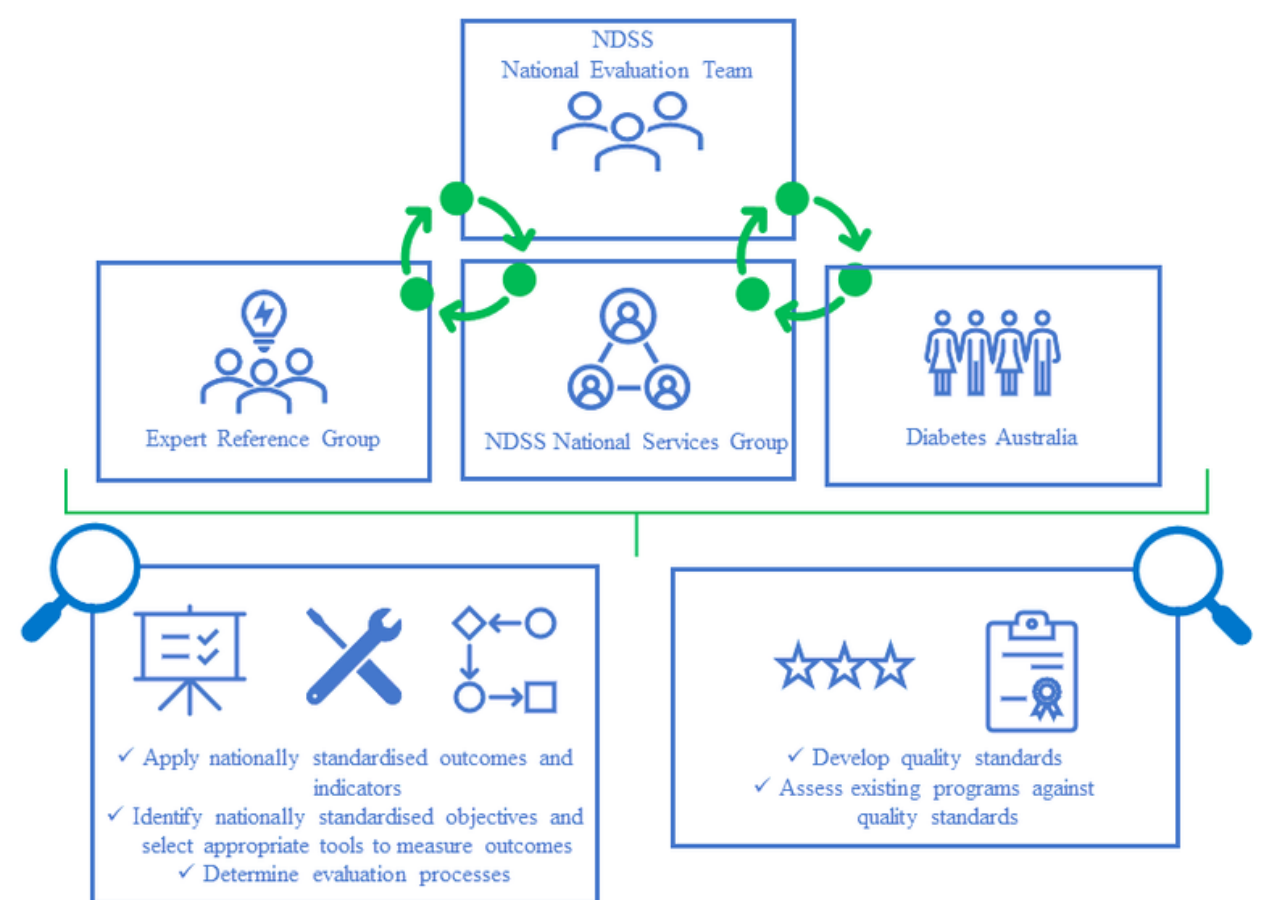

Figure 1 
Approach to develop the National Evaluation Framework

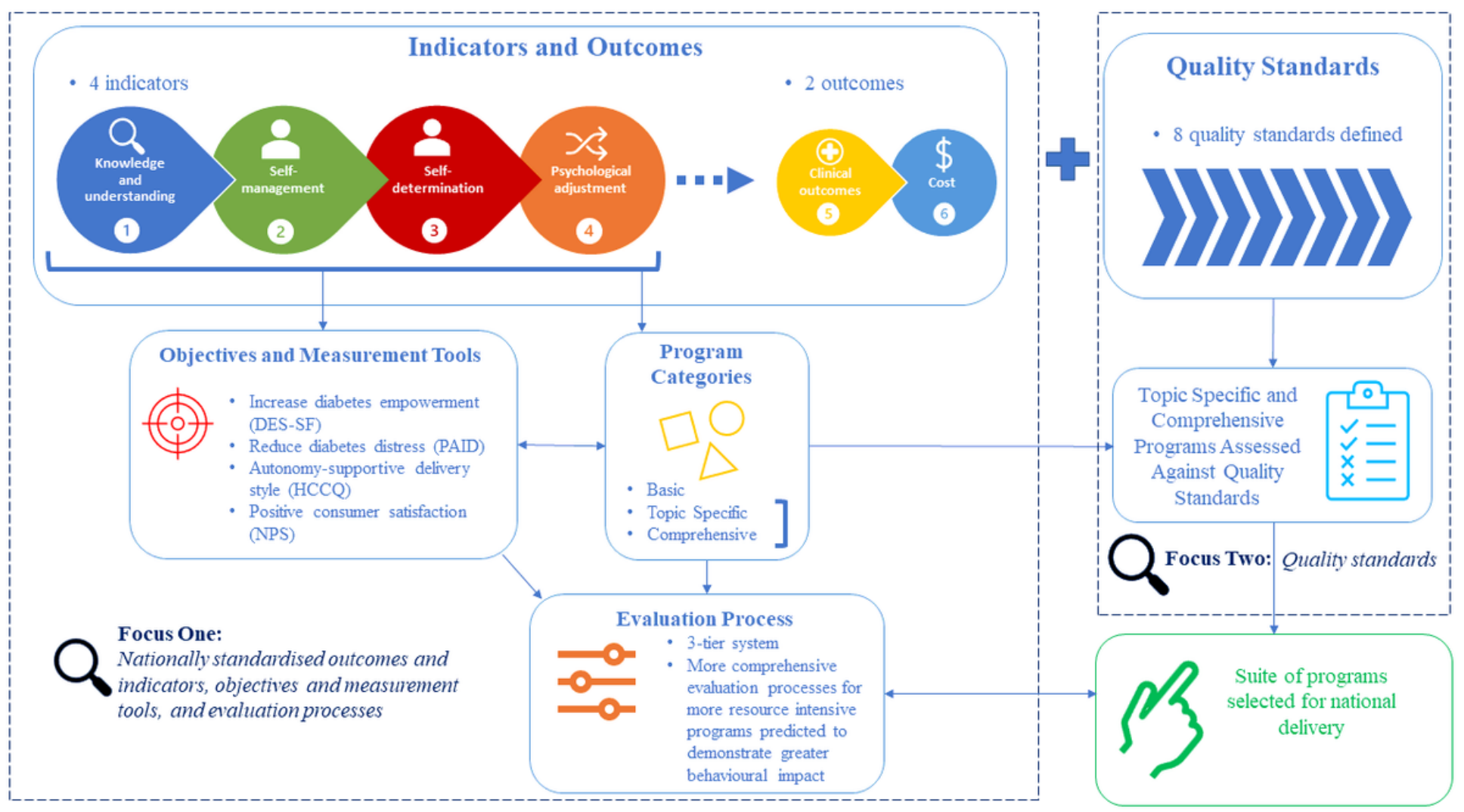

Figure 2

National Evaluation Framework

\section{Supplementary Files}

This is a list of supplementary files associated with this preprint. Click to download.

- AppendixANEFFramework.docx 